\title{
QUELS EFFETS DE LA PESTE PORCINE AFRICAINE SUR LES FILIÈRES PORCINES DANS LE MONDE ET LES AUTRES FILIÈRES ANIMALES?
}

\author{
WHICH CONSEQUENCES OF AFRICAN SWINE FEVER ON THE GLOBAL \\ SWINE INDUSTRY AND OTHER ANIMAL INDUSTRIES?
}

Par Boris DUFLOT ${ }^{1}$

(Communication présentée le 3 février 2021, note acceptée le 10 mai 2021)

\begin{abstract}
RÉSUMÉ
La PPA a occasionné une chute importante de la production chinoise de porc entre 2018 et 2020 . Pour combler ce déficit, la Chine a très nettement augmenté ses importations de viandes en 2019 et 2020, renforçant ainsi les tendances de croissance des exportations européennes et des Amériques et tirant les prix des viandes vers le haut dans ces régions. Cependant, dès le début de 2020, la COVID-19 a bouleversé les débouchés habituels des produits carnés sur ces marchés domestiques et à l'export, et provoqué des perturbations d'activité dans les industries des viandes, avec un effet dépressif sur les prix. Par ailleurs, l'émergence de la PPA en Allemagne en septembre 2020 a également fortement entravé les possibilités d'exportations européennes de porc. Les deux épidémies de PPA et COVID-19 mettent en évidence l'importance de la sécurisation sanitaire pour le développement économique et la résilience des filières animales.
\end{abstract}

Mots-clés : Marchés internationaux, Porc, Chine, Union européenne, Peste porcine africaine, Covid-19

\begin{abstract}
-ABSTRACT
ASF caused a significant drop in Chinese pork production between 2018 and 2020. To make up for this deficit, China significantly increased its meat imports in 2019 and 2020, thus strengthening the growth trend of European and American exports and driving up meat prices in these regions. However, from the start of 2020, COVID-19 disrupted the usual marketing channels for meat products in these domestic markets and in export markets, and caused disruption in the meat industries, with a depressive effect on prices. In addition, the emergence of ASF in Germany in September 2020 also severely hampered the possibilities for European pork exports. The two ASF and COVID-19 epidemics highlight the importance of health security for the economic development and the resilience of the animal sector.

Key-Words: International markets, Pig, Pork, China, European Union, African Swine fever, Covid-19
\end{abstract}

1. Directeur du Pôle Economie de I'IFIP - Institut du Porc, délégué général d'ABCIS. IFIP ; La Motte au Vicomte BP 35104 ; 35651 Le Rheu cedex. Courriel : boris.duflot@ifip.asso.fr 


\section{INTRODUCTION}

La PPA a occasionné une chute importante de l'offre mondiale de produits carnés à partir de la fin 2018. La baisse de production de porc en Chine entre 2018 et 2020 dépasse sans doute 30\% (16 Mt), selon les estimations de l'USDA (USDA). Par ailleurs, la production d'autres pays asiatiques (Vietnam, Philippines), qui comptent dans le bilan mondial, s'est contractée sensiblement sous l'effet de la maladie. Même si la production européenne atteint des sommets historiques, elle est quasi stationnaire à 24 Mt de 2018 à 2020 (Eurostat). Les Amériques (en particulier, les États-Unis et le Brésil ont en revanche poursuivi leur croissance durant cette période (USDA). Au final, le bilan porcin mondial se contracte au minimum de 13\% entre 2018 et 2020.

\section{DES BESOINS CHINOIS DANS TOUTES LES VIANDES}

Malgré les très forts investissements chinois dans de nouveaux complexes d'élevage, la récupération de la production nationale et, par extension mondiale, sera lente et probablement partielle. En effet, les substitutions entre viandes, en Chine et à l'échelle mondiale, se sont accélérées avec la PPA et laissent des traces durables. La production chinoise de volailles, encore inférieure de 60\% à celle du porc, a augmenté de $31 \%$ entre 2018 et 2020 (Hercule, 2020), et d'autres zones du monde poursuivent leur croissance, comme les États-Unis et surtout le Brésil. Plus limitée est la substitution par le bœuf, production et consommation minoritaires en Chine. Toutefois, la demande chinoise de viande bovine à l'import croît fortement et fait le jeu des exportateurs sud-américains et océaniens.

Face au déficit de viande porcine, la Chine a très nettement augmenté ses importations de toutes les viandes en 2019 et 2020, les circuits d'approvisionnement étant déjà activés depuis plusieurs années. Les importations de porc ont crû de $+175 \%$ entre 2018 et 2020 , celles de volailles de $+48 \%$ et de bouf $+32 \%$, soit $+79 \%$ pour les trois viandes cumulées (Husson \& Chaumet, 2020). Avec des impacts majoritairement localisés en Chine, la PPA a initialement renforcé les tendances de croissance des exportations de l'Europe, de l'Amérique du Nord et du Sud (Husson, 2019; Van Ferneij, 2020). Les effets sur les prix dans les bassins exportateurs ont été notables, notamment en Europe avec une hausse de 30\% pour le porc au second semestre 2019 par rapport à l'année précédente. La répartition des marges dans les filières s'est fortement modifiée, à l'avantage des éleveurs avec la montée du prix du porc, et au détriment des transformateurs, pris en étau entre la montée des prix des pièces de découpe de porc et une relative stabilité des prix à la consommation.

\section{LA COVID-19 MODIFIE L'IMPACT DE LA PPA SUR LES ÉCHANGES COMMERCIAUX}

L'ouverture franche de la fenêtre d'importations chinoises laissait augurer pour l'Europe et les Amériques plusieurs années de conjoncture favorable et d'investissements dédiés à l'exportation de viandes, que ce soit en élevage dans les zones de produc- tion où leur développement est envisageable, mais aussi en abattage-découpe et capacités de congélation (Van Ferneij, 2021). Cependant, dès le début 2020, la COVID-19 a chamboulé les flux commerciaux à l'intérieur des principales zones de production et de consommation mondiales. Les circuits de distribution et habitudes des consommateurs ont été perturbés, avec une forte baisse d'activité en restauration commerciale et collective et une hausse d'activité des circuits de la consommation à domicile comme les grandes surfaces et le e-commerce (Diot \& Duflot, 2021 ; FranceAgriMer, 2021). Les produits consommés, leur conditionnement, les opérateurs ont connu de forts changements, soit à la hausse, soit à la baisse.

De nombreux travailleurs des usines de la filière viandes ont été touchés par la COVID, aussi bien en Europe qu'aux Amériques. Ces usines recourent souvent à la main-d'œuvre étrangère et les mouvements de personnels entre pays ont été rendus plus difficiles avec la COVID. La réduction des capacités de production par manque de main-d'œuvre a limité les abattages, occasionnant des excédents de cheptel en élevage et à certains moments une rareté de l'offre de viande dans les étals, en particulier aux États-Unis (Husson et al. 2020). En Allemagne, la COVID a fermé durant plusieurs semaines des abattoirs parmi les plus grands d'Europe et a ravivé l'attention portée aux conditions de vie et de travail des ouvriers d'abattoirs, nombreux à souffrir de la maladie. Le plus grand opérateur, Tönnies, a lancé en réaction un plan de prévention sanitaire et d'amélioration des conditions de travail et d'hébergement de ses travailleurs. L'opinion et les pouvoirs publics avaient déjà à maintes reprises pointé du doigt la déresponsabilisation des abatteurs en raison du recours massif à la sous-traitance du travail. Cette pratique sera interdite à compter de janvier 2021.

Les échanges transcontinentaux ont également été affectés par des perturbations logistiques et le renforcement des contrôles sanitaires, atténuant fortement l'effet de la PPA sur les prix. L'extension de la PPA en Europe (Pologne dès 2014, nombreux pays de l'Est de l'UE touchés, Belgique dès septembre 2018, Allemagne à partir de septembre 2020) limite les possibilités d'exportations européennes de produits du porc. La Chine, la Corée du Sud, le Japon, principales destinations à l'export du porc européen et américain interdisent pour le moment les importations de produits du porc à partir du moment où des animaux, même de la faune sauvage et à distance des élevages, ont été touchés par la PPA.

\section{LA PPA EN EUROPE REBAT LES CARTES ENTRE EXPORTATEURS}

La reconnaissance d'un zonage sanitaire par les autorités chinoises, en cours de négociation entre la France et la Chine, rendrait l'Europe moins vulnérable à une extension future de la PPA. Le zonage est d'importance particulière pour la France, car sa production est très inégalement répartie sur le territoire et majoritairement située à l'ouest, à distance des foyers existants. En outre, les pays tiers représentent une bonne part des exportations françaises, plus de 50\% en 2020. La définition de compartiments sanitaires, ce qui serait nouveau pour l'espèce porcine en Europe, est également à l'étude. De tels compartiments, à 
l'échelle d'entreprises ou de groupes ayant défini des procédures sanitaires et de biosécurité communes, permettraient de maintenir des flux à l'export même dans les zones où des animaux sont infectés par la PPA. Ceci serait utile dans la mesure où l'avancée de la PPA en Europe risque d'être inéluctable, faute de vaccins et de mesures de restrictions spatiales infaillibles.

La PPA en Allemagne s'est matérialisée par une forte baisse de prix du porc dans ce pays, une baisse des importations d'animaux vivants et de produits intermédiaires de ses pays voisins, en particulier les porcelets venant du Danemark et des Pays-Bas. Les prix de ces produits ont chuté au dernier trimestre 2020. Les perspectives de production sont pessimistes en Allemagne, aux Pays-Bas et au Danemark. La PPA en Allemagne s'ajoute aux autres causes que sont le renforcement des objectifs environnementaux et la difficulté de recruter des éleveurs, exploitants et salariés.

L'Espagne profite au contraire de son accès privilégié aux marchés asiatiques. Après de très forts investissements en élevage et abattage-découpe ces dernières années, ses exportations ont crû de $21 \%$ en 2020, grâce à une croissance de $111 \%$ vers la Chine. Avec des filières intégrées verticalement, une capacité à développer l'élevage dans l'ensemble du pays et des démarches commerciales coordonnées et offensives, l'Espagne s'est montrée très réactive à combler l'appétit chinois.

\section{CONCLUSION}

Tout comme la crise COVID-19 l'a fait à l'échelle de l'ensemble de l'économie, la propagation de la PPA, en Asie comme en Europe, a remis au centre du jeu la gestion du risque sanitaire comme facteur de résilience et de performance des filières porcines. La PPA exerce un test de résistance et d'adaptation pour les élevages et les filières porcines. Dans ce contexte, les atouts des filières porcines résident dans leur capacité à saisir des opportunités à l'export aussi bien qu'à se protéger des aléas économiques et à mettre en œuvre d'efficaces mesures de protection sanitaire.

\section{BIBLIOGRAPHIE}

- Eurostat, office statistique de l'Union européenne. Tableaux sur la production de produits de l'élevage en dans l'UE. Disponible à https://appsso.e urostat.ec.europa.eu/nui/show.do?d ataset $=$ apro $\mathrm{mt}$ pwgtm\&lang $=\mathrm{fr}$ (Consulté le 10/05/2021).

- FranceAgriMer. "L'impact de la crise de la COVID-19 sur la consommation alimentaire en France : parenthèse, accélérateur ou élément de rupture de tendances ? ". 2020. Disponible à https://www.franceagrimer.fr/f am/content/download/65172/doc ument/15 CONSO $\% 20$ COVID\% 20 Impact $\% 20$ tendances vf.pdf?ve rsion=4 (Consulté le 10/05/2021).

- Diot V, Duflot B. Impact de la crise Covid-19 sur la consommation et la distribution des produits du porc en France. Journées Recherche Porcine
2021, $53: 297-302$.

- Hercule J, La viande de volaille, baromètre des crises sanitaires en Chine. 15/04/2020. Disponible à https://abcis.com/2020/04/15/laviande-de-volaille-barometredes-crises-sanitaires-en-chine/ (Consulté le 10/05/2021).

- Husson E. Commerce mondial du porc en 2019 : la Chine rafle le porc européen. Baromètre Porc 2020, 507 : 8

- Husson E, Chaumet JM. Importations chinoises record de produits porcins au printemps 2020. La Lettre Chine_ABCIS, 31/07/2020. Disponible à https://abcis.com/2020 /07/31/importations-chinoises-record -de-produits-porcins-au-printemps -2020/(Consulté le 10/05/2021).

- Husson E, Diot V, Sailley M. Covid-19 : face aux incertitudes, le cours du porc est sous pression. Note conjoncturelle IFIP, 12/05/2020, 3 pages. Disponible à https://www.ifip.asso.fr/fr/content/ covid-19-face-aux-incertitudes-lecours-du-porc-est-sous-pression-note -conjoncture-ifip-0 (Consulté le 10/05/2021)

- USDA, United States Department of Agriculture, World Agricultural Supply and Demand Estimates. Disponible à https://www.usda.gov/oce/commodity Lwasde (Consulté le 10/05/2021).

- Van Ferneij JP. La Fièvre Porcine Africaine rebat les cartes de la production porcine mondiale. Baromètre Porc 2020, $510: 8$.

- Van Ferneij JP, Duflot B, 2021. Des filières porcines qui s'internationalisent dans un contexte pourtant promoteur d'autonomie. Journées Recherche Porcine 2021, 53 : 309-314. 